\title{
Energy extremals and Nonlinear Stability in a Variational theory of a Coupled Barotropic Flow - Rotating Solid Sphere System
}

\author{
Chjan C. Lim \\ Mathematical Sciences, RPI, Troy, NY 12180, USA \\ email: limc@rpi.edu
}

To the memory of Lim Fu Mei and Chong Yat Ying

June 8, 2018

\begin{abstract}
A new variational principle - extremizing the fixed frame kinetic energy under constant relative enstrophy - for a coupled barotropic flow - rotating solid sphere system is introduced with the following consequences. In particular, angular momentum is transfered between the fluid and the solid sphere through a modelled torque mechanism. The fluid's angular momentum is therefore not fixed but only bounded by the relative enstrophy, as is required of any model that supports super-rotation.

The main results are: At any rate of spin $\Omega$ and relative enstrophy, the unique global energy maximizer for fixed relative enstrophy corresponds to solid-body super-rotation; the counter-rotating solidbody flow state is a constrained energy minimum provided the relative enstrophy is small enough, otherwise, it is a saddle point.

For all energy below a threshold value which depends on the relative enstrophy and solid spin $\Omega$, the constrained energy extremals
\end{abstract}


consist of only minimizers and saddles in the form of counter-rotating states. Only when the energy exceeds this threshold value can prorotating states arise as global maximizers.

Unlike the standard barotropic vorticity model which conserves angular momentum of the fluid, the counter-rotating state is rigorously shown to be nonlinearly stable only when it is a local constrained minima. The global constrained maximizer corresponding to superrotation is always nonlinearly stable. 


\section{Introduction}

The main aim of this paper is to present the simplest possible formulation and rigorous analysis of a theory for geophysical flow coupled to an infinitely massive rotating sphere, which exhibits characteristics of super and subrotation. Although the General Circulation Model (GCM) simulations reproduced some aspects of super-rotation such as those on Venus and Titan, a rigorous theory which can be analysed in classical mathematical terms, is lacking. This is the motivation which guides our approach here. We therefore propose a mathematically precise constrained variational problem in terms of the pseudo energy (rest frame kinetic energy) of a coupled barotropic flow on a rotating sphere, in which the only explicit constraints are relative enstrophy and zero total circulation. Unlike previous work, the angular momentum of the fluid is not fixed.

As the most promising phenomenological theory to date is one where the rotating atmosphere exchanges angular momentum with the planetary surface through a complex torque mechanism mediated by Hadley cells, we propose a variational theory to find and analyze the nonlinear stability of steady-states of the coupled fluid - sphere system. The coupled system is a conservative or non-dissipative model in the sense that the energy and angular momentum of the combined fluid-sphere system are separately conserved in time. We will not go into the details of standard arguments for the validity of an inviscid assumption for the interior flow in the context of large scale geophysical flows. Suffice it to say at this point that the role of viscosity is restricted to very thin Ekman boundary layers, and is within that part of the coupled fluid-sphere system modelled by the complex torque mechanism.

The main results of this approach is that, at any rate of spin $\Omega$ and relative enstrophy $Q_{r e l}$, the constrained global energy maximum for fixed relative enstrophy corresponds to solid-body rotation, $w_{\text {Max }}^{0}\left(Q_{\text {rel }}\right)=\sqrt{Q_{\text {rel }}} \psi_{10}$, in the direction of $\Omega$. Another solution, the counter-rotating steady state $w_{\text {min }}^{0}\left(Q_{\text {rel }}\right)=-\sqrt{Q_{\text {rel }}} \psi_{10}$, is a constrained global energy minimum provided the relative enstrophy is small enough, that is, $Q_{\text {rel }}<\Omega^{2} C^{2}$ where $C=\| \cos \theta$ $\|_{2}$. If $\Omega^{2} C^{2}<Q_{r e l}<4 \Omega^{2} C^{2}$, then $w_{\min }^{0}\left(Q_{r e l}\right)$ is a saddle point. And if $Q_{\text {rel }}>4 \Omega^{2} C^{2}$, then $w_{\min }^{0}\left(Q_{r e l}\right)$ is again a saddle point, but in the restricted sense that it is a constrained local energy maximum in all eigen-directions 
except $\psi_{1, \pm 1} ; w_{\min }^{0}\left(Q_{r e l}\right)$ is a constrained local energy minimum in the projection onto $\operatorname{span}\left(\psi_{10}, \psi_{1, \pm 1}\right)$ where the $\psi_{1, \pm 1}$ eigenmodes are relative vorticity patterns associated with the tilt instability.

The main result can be viewed another way. For all energy $H$ below a threshold value $H_{c}$ which depends on the relative enstrophy $Q_{r e l}$ and spin $\Omega$, the constrained energy extremals consist of only minima and saddles in the form of counter-rotating states, $-\sqrt{Q_{\text {rel }}} \psi_{10}$. Only when the energy exceeds this threshold value $H_{c}$, can pro-rotating states, $\sqrt{Q_{\text {rel }}} \psi_{10}$ arise as constrained global energy maxima.

It is also useful to view the approach in this paper in the light of a vertically coarse-grained variational analysis of the steady states of the barotropic component of fully stratified rotating flows coupled to a massive rotating sphere. We recall another robust connection between the time-independent variational theory presented and dynamic initial value problems for damped driven quasi-geostrophic flows: The Principle of Selective Decay or Minimum enstrophy states that, for suitable initial data, the long time asymptotics of the 2D Navier-Stokes equations - damped and forced on a doubly-periodic domain (and by extension the sphere) - is characterized by a decreasing enstrophy to energy ratio [6]. These Minimum Enstrophy flows are characterized by special steady states which are the solid body rotational flows in the case of the sphere, by virtue of an application of Poincare's Inequality 23. In 1953, Fjortoft 8 used a spherical harmonics expansion to show that, for dissipative barotropic flows, because the enstrophy spectrum is related to the energy spectrum by $Z(l, t)=l(l+1) E(l, t)$, the energy fluxes towards low wavenumbers $l$ while enstrophy fluxes towards high $l$ modes. His work is influential in bringing forth the concepts of the inverse cascade of energy and forward cascade of enstrophy that are closely related to Selective Decay.

Indeed, an inviscid asymptotic formulation of the Principle of Minimum Enstrophy yields a variational problem that is the dual of the one in this paper, namely, extremize enstrophy at fixed values of the total kinetic energy of flow without fixing the angular momentum of the fluid. The corresponding extremals including states of minimum enstrophy are again solid-body rotating flows. Leith [9] formulated a related method precisely by looking for constrained enstrophy extremals in vortex dynamics with fixed angular momentum of the fluid.

Stability results for barotropic flows are discussed in Fjortoft [7, Tung [22], and Shepherd et al [3], [10] amongst many others. Fjortoft [7] first used integral theorems to argue that solid-body rotational flows are always stable 
in the context of the Hamiltonian PDE known as the barotropic vorticity equaton (BVE). The discrepancy between his result and those reported here - the retrograde solid-body flow is always a stable steady-state in the BVE but has only conditional nonlinear stability in the coupled barotropic flow - rotating solid sphere system - is partly due to the simple but vital fact that the fluid's angular momentum is allowed to vary in the latter. We will elaborate on this point below.

Arnold [11], [12 formalized this body of results known as the energyCasimir method, and proved that they work at finite amplitudes, that is in a nonlinear way. According to an argument of Shepherd in his refined application of the energy-Casimir method to the BVE [1], the Lyapunov function method fails precisely when the planetary spin $\Omega$ is small - the proof of the nonlinear stability of solid-body flow states in the BVE breaks down when the spin is small or zero. See also the note by Wirosoetisno and Shepherd [10] which proved that there are two important cases in 2D Euler flows where the Arnold stability method fails, and one of them is the non-rotating sphere. Our result on the conditional instability of the retrograde (east to west) solid-body flow and the nonlinear stability of the prograde solid-body flow is partially compatible with Shepherd et al because we proved instability of the retrograde flow state only when the planetary spin $\Omega$ is smaller than $\sqrt{Q_{r e l} / C^{2}}$, and because our result also states that the prograde rigid rotation state is nonlinearly stable even when the planetary spin is relatively small. Again the discrepancy between our results and those of Shepherd et al can be traced to the fact that the coupled barotropic fluid sphere model is not - unlike the BVE - a Hamiltonian PDE and angular momentum of the fluid is not conserved.

Indeed, the correct variational theory proposed here is related to the zero temperature version of the statistical mechanics energy - relative enstrophy theory given in [2], [16]. There as here, the underlying model - a coupled fluid sphere system - cannot be represented by a Hamiltonian or a Lagrangian. Therefore, there is no local in time equation of motion for the underlying model but rather a generalized variational principle operating in overall phase space determines its symmetries and dynamical properties. This variational theory and corresponding statistical mechanics theory comprise a new example of Feynman's generalized Least Action Principle that is applicable to non-local situations which do not have a Hamiltonian or Lagrangian formulation. In this setting, we propose a non-Hamiltonian energy 
functional that plays a principal role in the theory presented here and, as the integrand in the action of a partition function (or path-integral) 2], in the corresponding statistical mechanics theory as well.

This statistical mechanics theory predicts that the macrostates with extremal internal energy - highly organized, maximally solid-body flow states - are invariably associated with low Shannon entropy, and are therefore the most probable states when the absolute value of the statistical temperature is small. On the other hand, when the temperatures have large absolute values, the most probable macrostates - disordered flow states with little or zero net angular momentum - have intermediate values of internal energy and high Shannon entropy [2]

In section 2 we give properties of the model, as useful background for the rest of the paper. Section 3 describes the energy-enstrophy class of variational formulations for the coupled fluid - sphere model. Section 4 gives the constrained optimization of flow energy, $H$ on fixed relative enstrophy manifolds and section 5 describes the physical characteristics of the constrained energy extremals that arise in the model. Section 6 gives the nonlinear stability results. Section 7 concerns physical consequences and concluding remarks.

\section{Coupled Barotropic Fluid - Rotating solid Sphere Model.}

Consider the system consisting of a rotating massive rigid sphere of radius $R$, enveloped by a thin shell of non-divergent barotropic fluid. The barotropic flow is assumed to be inviscid, apart from an ability to exchange angular momentum and kinetic energy with the infinitely massive solid sphere through a complex torque mechanism. We also assume that the fluid is in radiation balance and there is no net energy gain or loss from insolation. This provides a crude model of the complex planet - atmosphere interactions, including the enigmatic torque mechanism responsible for the phenomenon of atmospheric super-rotation - one of the main applications motivating this work.

For a geophysical flow problem concerning super-rotation on a spherical surface there is little doubt that one of the key parameters is angular momentum of the fluid. In principle, the total angular momentum of the fluid and solid sphere is a conserved quantity but by taking the sphere to have infinite mass, the active part of the model is just the fluid which relaxes by 
exchanging angular momentum with an infinite reservoir. It is also clear that a $2 \mathrm{~d}$ geophysical relaxation problem such as this one will involve energy and enstrophy. The rest frame energy of the fluid and sphere is conserved. Since we have assumed the mass of the solid sphere to be infinite, we need only keep track of the kinetic energy of the barotropic fluid - in the non-divergent case, there is no gravitational potential energy in the fluid because it has uniform thickness and density, and its upper surface is a rigid lid.

In a nutshell, we need to find a suitable set of constraints for the obvious choice of objective functional, namely rest frame kinetic energy of flow in the coupled model. The choice of auxillary conditions or constraints is not apriori obvious, as different choices can have slightly different physical consequences 24].

We will use spherical coordinates - $\cos \theta$ where $\theta$ is the colatitude and longitude $\phi$. The total vorticity is given by

$$
q(t ; \cos \theta, \phi)=\Delta \psi+2 \Omega \cos \theta
$$

where $2 \Omega \cos \theta$ is the planetary vorticity due to spin rate $\Omega$ and $w=\Delta \psi$ is the relative vorticity given in terms of a relative velocity stream function $\psi$ and $\Delta$ is the negative of the Laplace-Beltrami operator on the unit sphere $S^{2}$.

\subsection{Inverse of the Laplacian}

Key to the analysis in this paper is the inverse integral operator defined by

$$
G[f](x)=-\frac{1}{2} \int_{S^{2}} d x^{\prime} f\left(x^{\prime}\right) \ln \frac{1}{\left|x-x^{\prime}\right|}
$$

on $S^{2}$. In terms of $G$, the solution of

$$
\Delta \psi=f(x)
$$

is given by

$$
\begin{aligned}
\psi(x) & =\Delta^{-1}(f)=G[f](x) \\
& =-\frac{1}{2} \int_{S^{2}} d x^{\prime} f\left(x^{\prime}\right) \ln \frac{1}{\left|x-x^{\prime}\right|}
\end{aligned}
$$




\subsection{Eigenfunctions of $G$}

The variational analysis of this problem is based on the fact that there is an orthonormal basis for $L^{2}\left(S^{2}\right)$ of eigenfunctions of the Laplace-Beltrami operator. And $G$ being the inverse of $\Delta$, its eigenfunctions consist of the spherical harmonics,

$$
\begin{aligned}
\psi_{l m}, m & =-l, \ldots, l \\
\text { with eigenvalue } \lambda_{l m} & =-1 /[l(l+1)] .
\end{aligned}
$$

Thus, a relative vorticity field, by Stokes theorem, has expansion

$$
w(x)=\sum_{l \geq 1, m} \alpha_{l m} \psi_{l m}(x) .
$$

A key property that will be established later is that the mode $\alpha_{10} \psi_{10}(x)$ contains all the angular momentum in the relative flow with respect to the frame rotating at the fixed angular velocity $\Omega$ of the sphere.

\subsection{Physical quantities of the coupled barotropic vor- ticity model}

The rest frame kinetic energy of the fluid expressed in a frame that is rotating at the angular velocity of the solid sphere is

$$
\begin{aligned}
H_{T}[q] & =\frac{1}{2} \int_{S^{2}} d x\left[\left(u_{r}+u_{p}\right)^{2}+v_{r}^{2}\right] \\
& =\frac{1}{2} \int_{S^{2}} d x\left[\left(u_{r}^{2}+v_{r}^{2}\right)+2 u_{r} u_{p}\right]+\frac{1}{2} \int_{S^{2}} d x u_{p}^{2} \\
& =-\frac{1}{2} \int_{S^{2}} d x \psi q+\frac{1}{2} \int_{S^{2}} d x u_{p}^{2}
\end{aligned}
$$

where $u_{r}, v_{r}$ are the zonal and meridional components of the relative velocity, $u_{p}$ is the zonal component of the planetary velocity (the meridional component being zero since planetary vorticity is zonal), and $\psi$ is the stream function for the relative velocity.

Since the second term $\frac{1}{2} \int_{S^{2}} d x u_{p}^{2}$ is fixed for a given spin rate $\Omega$, it is convenient to work with the pseudo-energy as the energy functional for the model,

$$
H[w]=-\frac{1}{2} \int_{S^{2}} d x \psi q=-\frac{1}{2} \int d x \psi(x)[w(x)+2 \Omega \cos \theta]
$$




$$
\begin{aligned}
& =-\frac{1}{2} \int d x \psi(x) w(x)-\Omega \int d x \psi(x) \cos \theta \\
& =-\frac{1}{2}\langle w, G[w]\rangle-\Omega C\left\langle\psi_{10}, G[w]\right\rangle
\end{aligned}
$$

where $C=\|\cos \theta\|_{2}$. Later, it will be convenient to perform one more minor adjustment of the energy functional in Lemma 3 to turn it into a positive definite quadratic form in the Fourier coefficients of the spectral expansion of the relative vorticity $w$ in the spherical harmonics $\psi_{l m}$. The following calculation using the self-adjoint and eigenvalue properties of the integral operator $G$,

$$
\begin{aligned}
-C\left\langle\psi_{10}, G[w]\right\rangle & =-C\left\langle G\left[\psi_{10}\right], w\right\rangle \\
& =\frac{C}{2}\left\langle\psi_{10}, w\right\rangle \\
& =\frac{1}{2} \int_{S^{2}} d x w(x) \cos \theta(x),
\end{aligned}
$$

shows that

$$
\Lambda[w] \equiv-C\left\langle\psi_{10}, G[w]\right\rangle=\frac{\alpha_{10} C}{2}
$$

is the variable net angular momentum of the flow associated with the relative vorticity $w(x)$ (assuming unit mass density). Since

$$
E[w] \equiv-\frac{1}{2}\langle w, G[w]\rangle=-\frac{1}{2} \int d x \psi(x) w(x)
$$

is the kinetic energy of relative motion, this suggests that the pseudo-energy $H[q]=-\frac{1}{2} \int_{S^{2}} d x \psi q$ has the form

$$
H[q]=E[w]+\Omega \Lambda[w]
$$

of an energy-momentum functional used in standard variational methods for rotating problems. This is however only a coincidence.

A key difference between our approach and the standard variational analysis of rotating problems is that neither angular momentum $\Lambda[w]$ nor kinetic energy $H$ is fixed in our approach - the coefficient $\alpha_{10}$ in the expansion (3) is not fixed.

Total circulation in the model is fixed to be $\int q d x=\int w d x=0$, which is a direct consequence of Stokes theorem on a sphere and has nothing to do with the fact that the barotropic flow is assumed to be inviscid. It is easy 
to see that the kinetic energy functional $H$ is not well-defined without the further requirement of a constraint on the size of its argument, the relative vorticity field $w(x)$. A natural constraint for this quantity is therefore its square norm or relative enstrophy so as to carry out the variational analysis in the Hilbert space, $L^{2}\left(S^{2}\right)$. But before we settle on this constraint for the variational theory, we consider the total enstrophy of the flow,

$$
\Gamma[q]=\int_{S^{2}} d x q^{2}
$$

Expanding (cf. [1]),

$$
\begin{aligned}
\Gamma[q] & =\int_{S^{2}} d x q^{2}=\int_{S^{2}} d x[w+2 \Omega \cos \theta]^{2} \\
& =\int_{S^{2}} d x w^{2}+4 \Omega \int_{S^{2}} d x w \cos \theta+4 \Omega^{2} C^{2}
\end{aligned}
$$

we find that the last term is a conserved quantity because it is the square of the $L^{2}$ norm of the fixed planetary vorticity, the second term is proportional to the variable net angular momentum of the fluid (relative to the rotating frame), and the first term is the relative enstrophy.

From (44), we deduce that, unlike the BVE, at most one of the two quantities - total enstrophy and relative enstrophy - can be conserved in the coupled fluid-sphere model. In other words, if the total enstrophy is conserved then relative enstrophy is not because net angular momentum is not conserved; if on the other hand, relative enstrophy is conserved, then total enstrophy must change with net angular momentum. In fact it is not possible - from what is assumed in the model - to exclude the third possibility that neither the relative entrophy nor total enstrophy is conserved.

Thus, we see that our choice of the relative enstrophy constraint, although it is natural and required for a rigorous variational analysis, is not a consequence of its invariance. Instead the argument that to an extent justifies this choice is based on the Principle of Minimum Enstrophy. This Principle states that only the ratio of enstrophy to energy - not their separate values should be relevant to the analysis of a quasi-steady state in $2 \mathrm{~d}$ flows and this ratio should have the minimum allowed value for the given geometry. This is clearly dual and equivalent to characterizing quasi-steady states in $2 \mathrm{~d}$ flows in terms of their energy on iso-enstrophy manifolds.

The second term in (41) is equal to $4 \Omega$ times the variable angular momentum density of the relative fluid motion and has units of $\mathrm{m}^{4} / \mathrm{s}$. The physical 
angular momentum, given by

$$
\rho \int_{S^{2}} d x w \cos \theta=\rho\langle w, \cos \theta\rangle
$$

implies that the only mode in the eigenfunction expansion of $w$ that contributes to its net angular momentum is $\alpha_{10} \psi_{10}$ where $\psi_{10}=a \cos \theta$ is the first nontrivial spherical harmonic; it has the form of solid-body rotation vorticity.

All other moments of the vorticity $\int d x q^{n}$ are deemed less important in many physical theories of fluid motions including the absolute equilibrium statistical mechanics model [15] and the variational problems below 17. They are, however, not totally irrelevant [24].

\section{Energy-relative enstrophy variational the- ory}

One of the aim of this approach is to answer the question: For which relative vorticity field $w(x)$ is the fluid's rest frame kinetic energy extremal under the single additional constraint that the square-norm of $w(x)$ or relative enstrophy is fixed? It turns out to be physically relevant that the $w_{0}(x)$ that extremizes $H[q]$ also maximizes the net angular momentum $\Lambda[w]$ since then, these extremal steady states are super or sub-rotating according to the sign of $\alpha_{10}$ in the expansion (3) of $w_{0}(x)$.

A similar analysis for the non-rotating case $(\Omega=0)$ shows below that $H[w]=E[w]$ is maximized under fixed enstrophy by pro-rotating solid-body flow $w_{0}(x)=\alpha_{10} \psi_{10}(x)$ for $\alpha_{10}>0$ which at the same time maximizes net angular momentum $\Lambda\left[w_{0}\right]$. This implies immediately the proposition:

Proposition: For fixed $\Omega>0, H[q]$ is maximized under fixed relative enstrophy by the solid - body flow $w_{0}(x)$ which also maximizes relative or net angular momentum $\Lambda\left[w_{0}\right]$.

What is not obvious and therefore requires the substantial analysis presented in this paper is the asymmetry between the energy maximizer and mnimizer - the counter-rotating solid-body flow $w_{1}(x)=\alpha_{10} \psi_{10}(x)$ for $\alpha_{10}<0$, corresponding to minimal angular momentum, and maximizing $E[w]$ (since the energy $E[w]$ is even in $w$ ), is a minimizer of $H[q]$ for values of relative enstrophy that are small compared to the rotation rate $\Omega$, but becomes a saddle point for larger values of relative enstrophy. 
Not only are the higher vorticity moments $\int d x q^{n}$ for $n>2$ ignored in this paper (cf. also [15]. We also do not constrain the total enstrophy

$$
\int_{S^{2}} d x w^{2}+4 \Omega \int_{S^{2}} d x w \cos \theta
$$

nor the angular momentum

$$
\int_{S^{2}} d x w \cos \theta
$$

The only constraint in this paper besides the zero total circulation condition,

$$
\int_{S^{2}} d x w=0
$$

is that the relative enstrophy,

$$
\int_{S^{2}} d x w^{2}=Q_{r e l}>0
$$

is fixed.

\subsection{Constraint $V_{\text {rel }}$}

We first analyze the physical consequences of the constraint $\|w\|_{2}=Q_{\text {rel }}>0$ in the definition of the subset

$$
V_{\text {rel }}=\left\{w \in L^{2}\left(S^{2}\right) \mid \Gamma_{\text {rel }}[w]=\|w\|_{2}=Q_{\text {rel }}>0, T C=\int_{S^{2}} d x w=0\right\},
$$

which leave the angular momentum (6) constrained only by an inequality. Fixing the relative enstrophy and relative circulation, this model allows in principle energy extrema which can have up to a maximum amount of super or sub-rotation. It has the interesting consequence that angular momentum is effectively constrained by an inequality.

Lemma 1: Let the circulation and relative enstrophy of $w \in L^{2}\left(S^{2}\right)$ be constrained by (7) and (8) respectively. Then, the angular momentum (6) is constrained by an inequality, that is,

$$
\left|\int_{S^{2}} d x w \cos \theta\right| \leq C \sqrt{Q_{r e l}}
$$

where $C>0$ is a constant that does not depend on $w$. 
Proof: By the Cauchy-Schwartz inequality [23], the result is proved with

$$
C^{2}=\int_{S^{2}}(\cos \theta)^{2} d x
$$

QED.

It is not surprising that solid-body rotation vorticity in the form of spherical harmonic $\psi_{10}=b \cos \theta$ is the only zero circulation relative vorticity field that maximizes angular momentum for given enstrophy. This is stated precisely below:

Lemma 2: The upper bound on the angular momentum in (9) is achieved only for relative vorticity field

$$
w=k \cos \theta
$$

where $k$ is a constant.

Proof: When $w=k \cos \theta$,

$$
\left|\int_{S^{2}} d x w \cos \theta\right|=|k|\left|\int_{S^{2}} d x \cos ^{2} \theta\right|=|k| C^{2}
$$

and

$$
\|w\|^{2}=\int_{S^{2}} d x w^{2}=k^{2} C^{2}=Q_{r e l}
$$

Thus,

$$
\left|\int_{S^{2}} d x w \cos \theta\right|=C \sqrt{Q_{r e l}}
$$

Conversely, if

$$
w=k \cos \theta+a \psi_{l m}
$$

where $l m \neq 10$ and $a \neq 0$, then

$$
\left|\int_{S^{2}} d x w \cos \theta\right|<C|| w \| \mid
$$

QED. 


\subsection{Augmented energy functionals}

The Lagrange Multiplier method for Hilbert space [21] gives necessary conditions for constrained extremals in the form of Euler-Lagrange equations. More importantly, the explicit form of the Lagrange Multipliers in terms of spin, energy and relative enstrophy, provides the detailed physical relationships that we seek in order to answer the questions raised. We also extend the Lagrange Multiplier method to give a geometrical proof of the existence and nonlinear stability of the constrained energy extremals where the constraint is fixed relative enstrophy.

The unconstrained optimization of the augmented energy-relative enstrophy functional arising from the Lagrange Multiplier method yield necessary conditions for extremals of the constrained optimization problems. Necessary conditions for the extremals of the augmented functionals are in turn given in terms of their Euler-Lagrange equations (or Gateaux derivative) which take the form of interesting inhomogeneous linear equations involving the inverse $G$ of the Laplace-Beltrami operator. Bifurcation values of the multipliers can be read off the spectrum of $G$. Extremals of the augmented energy functional typically change type for different regimes of the Lagrange multiplier values which are marked by bifurcation values.

The relative enstrophy constraint gives rise to the following constrained optimization problems:

$$
\begin{gathered}
\text { extremize } H[w] \text { on } \\
V_{\text {rel }}=\left\{w \in L^{2}\left(S^{2}\right) \mid \Gamma_{r e l}[w]=\|w\|_{2}=Q_{\text {rel }}>0, \quad \int_{S^{2}} d x w=0\right\}
\end{gathered}
$$

By the method of Lagrange Multipliers (see [21, 20]), the energy-relative enstrophy functional

$$
E_{\text {rel }}[w ; \Omega, R]=H[w]+\lambda \Gamma_{\text {rel }}[w]
$$

is the augmented objective functional for the unconstrained optimization problem coresponding to the above constrained optimization problem.

\subsection{Lagrange Multipliers}

The Euler-Lagrange Multiplier theorem states: Let $w_{0} \in V_{\text {rel }}$ be an extremal of $H[w]$. Then at least one of the following holds: 


$$
\text { (1) } \delta \Gamma_{r e l}\left(w_{0}\right)=0 \text {, }
$$

$$
\text { (2) } \delta E_{\text {rel }}\left(w_{0}\right)=\delta H\left(w_{0}\right)+\lambda \delta \Gamma_{\text {rel }}\left(w_{0}\right)=0 \text {. }
$$

The right way to use this theorem is: (a) find a set of relative vorticity $w \in V_{\text {rel }}$ which satisfies $\delta \Gamma_{r e l}(w)=0$, (b) find a second set of relative vorticity $w \in V_{\text {rel }}$ which satisfies $\delta H(w)+\lambda \delta \Gamma_{\text {rel }}(w)=0$, for some $\lambda$, (c) the set of constrained extremals of $H[w]$ is contained in the union of the sets in (a) and (b), and (d) the value of $\lambda$ is determined from the value of the fixed constant $\Gamma_{\text {rel }}[w]=Q_{\text {rel }}>0$.

In implementing this procedure we first compute the variation or Gateaux derivative

$$
\delta \Gamma_{r e l}(w, \Delta w)=2 \int_{S^{2}} w \Delta w d x .
$$

By choosing $\Delta w=w$, we show that this variation never vanishes for any $w$, i.e.,

$$
\text { (1) } \delta \Gamma_{\text {rel }}(w) \neq 0 \text {. }
$$

The Euler-Lagrange Multiplier theorem then states that any extremal $w_{0}$ of the constrained variational problem (11) and (12) must satisfy

$$
\text { (2) } \delta E_{r e l}\left(w_{0}\right)=\delta H\left(w_{0}\right)+\lambda \delta \Gamma_{r e l}\left(w_{0}\right)=0
$$

for some value of $\lambda$.

The vanishing of the Gateaux derivative of the augmented functional $E_{r e l}$ gives the Euler-Lagrange equation for the unconstrained problem, which is solved in the next section.

\section{Extremals of the augmented energy func- tional}

The analysis below will be based on the spherical harmonics (2) which are eigenfunctions of the Laplace-Beltrami operator on the sphere. In particular, the eigenfunction $\psi_{10}=a \cos \theta$ which has eigenvalue $\lambda_{10}=-2$, will play a 
special role in the characterization of the zonal steady states. Using the linearity of $G$, and the eigenfunction expansion

$$
w=\sum_{l \geq 1, m} \alpha_{l m} \psi_{l m}
$$

of relative vorticity $w$ satisfying $\int_{S^{2}} d x w=0$, the Hamiltonian functional is expanded in terms of the orthonormal spherical harmonics, to yield

$$
\begin{aligned}
H & =-\frac{1}{2}\langle w, G[w]\rangle-\Omega C\left\langle\psi_{10}, G[w]\right\rangle \\
& =-\frac{1}{2} \sum_{l \geq 1, m} \frac{\alpha_{l m}^{2}}{\lambda_{l m}}+\frac{1}{2} \Omega C \alpha_{10}
\end{aligned}
$$

It follows that the coupling between the relative motion and the planetary vorticity occurs through the eigenfunction $\psi_{10}$, which has the form of a vorticity pattern corresponding to solid-body rotational flow.

For obvious technical reasons, and without loss of generality, we will use the following lemma to change the Hamiltonian by a constant to a quadratic form.

Lemma 3: For fixed spin $\Omega>0$, the energy $H$ for $w$ satisfying

$$
\int_{S^{2}} d x w(x)=0
$$

is modulo the constant

$$
H_{0}=-\frac{1}{4} \Omega^{2} C^{2}
$$

equal to the positive definite quadratic form (which we will again denote by $H)$

$$
H=\frac{1}{4}\left[\alpha_{10}-(-\Omega C)\right]^{2}+\frac{1}{4}\left[\alpha_{11}^{2}+\alpha_{1-1}^{2}\right]-\frac{1}{2} \sum_{l>1, m} \frac{\alpha_{l m}^{2}}{\lambda_{l m}}
$$

Proof: By completing the square,

$$
H=-\frac{1}{2} \sum_{l \geq 1, m} \frac{\alpha_{l m}^{2}}{\lambda_{l m}}+\frac{1}{2} \Omega C \alpha_{10}
$$




$$
\begin{aligned}
& =\frac{1}{4} \alpha_{10}^{2}+\frac{1}{2} \Omega C \alpha_{10}-\frac{1}{2} \sum_{l \geq 1, m} \frac{\alpha_{l m}^{2}}{\lambda_{l m}} \\
& =\frac{1}{4}\left[\alpha_{10}^{2}+2 \Omega C \alpha_{10}+\Omega^{2} C^{2}\right]+\frac{1}{4}\left[\alpha_{11}^{2}+\alpha_{1-1}^{2}\right]-\frac{1}{2} \sum_{l>1, m} \frac{\alpha_{l m}^{2}}{\lambda_{l m}}-\frac{1}{4} \Omega^{2} C^{2} \\
& =\frac{1}{4}\left[\alpha_{10}-(-\Omega C)\right]^{2}+\frac{1}{4}\left[\alpha_{11}^{2}+\alpha_{1-1}^{2}\right]-\frac{1}{2} \sum_{l>1, m} \frac{\alpha_{l m}^{2}}{\lambda_{l m}}-\frac{1}{4} \Omega^{2} C^{2} \\
& \text { QED. }
\end{aligned}
$$

Since all the extremals $w^{0}$ below have the form $k \psi_{10}$ of solid-body rotation, it is useful to state the following result. The simple proof is left to the reader. Graphs of (18) and (19) are depicted in figures 1 and 2.

Lemma 4: The energy and relative enstrophy of the extremals $w^{0}=$ $\alpha_{10} \psi_{10}$ takes the form

$$
\begin{aligned}
H\left[\alpha_{10} \psi_{10}\right] & =\frac{1}{4}\left(\alpha_{10}+\Omega C\right)^{2} \\
Q_{r e l} & \equiv \Gamma_{r e l}\left[\alpha_{10} \psi_{10}\right]=\alpha_{10}^{2}
\end{aligned}
$$

Furthermore, for fixed $H$,

$$
Q_{r e l} \pm 2 \Omega C \sqrt{Q_{r e l}}+\Omega^{2} C^{2}=4 H
$$

with solutions

$$
Q_{r e l}=( \pm \Omega C+\sqrt{4 H})^{2}
$$

\subsection{Lagrange Multipliers}

This constrained variational model is based on the fixed relative enstrophy constraint $V_{\text {rel }}$. By a theorem in [21] on the Lagrange Multiplier method, the variational problem (11), (12) can be reformulated in terms of extremals of the augmented functional,

$$
\begin{aligned}
E_{r e l}[w ; \Omega] & =H[w]+\lambda_{r e l} \Gamma_{r e l}[w] \\
\Gamma_{r e l}[w] & =\int_{S^{2}} w^{2} d x
\end{aligned}
$$


Expanding $\Gamma_{r e l}[w]$ in terms of (14) and combining with (17) yields

$$
E_{r e l}[w ; \Omega]=\frac{1}{4}\left[\alpha_{10}-(-\Omega C)\right]^{2}+\frac{1}{4}\left[\alpha_{11}^{2}+\alpha_{1-1}^{2}\right]-\frac{1}{2} \sum_{l>1, m} \frac{\alpha_{l m}^{2}}{\lambda_{l m}}+\lambda_{r e l} \sum_{l \geq 1, m} \alpha_{l m}^{2} .
$$

Taking the Gateaux derivative of $E_{r e l}[w ; \Omega]$ wrt $w$ gives the Euler-Lagrange equation,

$$
\left[G-2 \lambda_{r e l}\right]\left(w^{0}\right)=\frac{1}{2} \Omega C \psi_{10} .
$$

By the Fredholm Alternative [23, equation (22) either (a) has solutions for all values of the right hand side, or (b) has infinite number of solutions when the right hand side is orthogonal to the kernel of $\left[G-2 \lambda_{\text {rel }}\right]$. All the eigenvalues of $G$ are negative and form an increasing sequence, $\lambda_{l m}^{-1}$. There are several subcases, namely, (1) $\lambda_{\text {rel }} \in\left(-\infty,-\frac{1}{4}\right)$, (2) $\lambda_{\text {rel }} \in\left(-\frac{1}{4}, \infty\right), \lambda_{\text {rel }}$ $\neq-\frac{1}{2 l(l+1)}$ and $(3) \lambda_{\text {rel }}=-\frac{1}{2 l(l+1)} \in\left[-\frac{1}{4}, 0\right)$, which fall into the two broader classes that depend on whether or not the kernel of $\left[G-2 \lambda_{r e l}\right]$ is trivial.

Although the results in the following theorem clearly have physical significance, they are nonetheless easy to establish because the Euler-Lagrange equation (22) is a linear equation. Figure 4 shows plots of (23) and (24).

\section{Theorem 5:}

(1) Only when $\lambda_{\text {rel }} \in\left(-\infty,-\frac{1}{4}\right)$, can extremal relative vorticity in the form of solid-body rotation in the same direction as planetary vorticity arise

(2) For $\lambda_{\text {rel }} \in\left(-\frac{1}{4}, \infty\right), \lambda_{\text {rel }} \neq-\frac{1}{2 l(l+1)}$, the extremal vorticity (if it exists), is solid-body rotation in the opposite direction as planetary vorticity;

(3) Only when $\lambda_{\text {rel }}=-\frac{1}{2 l(l+1)} \in\left[-\frac{1}{4}, 0\right)$, can higher spherical harmonics $\psi_{l m}, l m \neq 10$, contribute to the extremal vorticity.

The proof is divided into two natural subcases, namely, (a) when the kernel of $\left[G-2 \lambda_{r e l}\right]$ is empty, and (b) when it is not.

(a i) $\lambda_{\text {rel }} \in\left(-\infty,-\frac{1}{4}\right)$

In this regime, the kernel of $\left[G-2 \lambda_{r e l}\right]$ is also empty, which implies the results:

$$
\text { for } \lambda_{r e l} \in\left(-\infty,-\frac{1}{4}\right), w^{0}=-\frac{\Omega C}{2\left(\frac{1}{2}+2 \lambda_{r e l}\right)} \psi_{10} \text {; }
$$

and,

$$
w^{0}=k \psi_{10} \text { with } k \rightarrow 0^{+} \text {as } \lambda_{\text {rel }} \rightarrow-\infty
$$




$$
w^{0}=k \psi_{10} \text { with } k \rightarrow \infty \text { as } \lambda_{\text {rel }} \rightarrow-\frac{1}{4} .
$$

Thus, there is a pole-like singularity at $\lambda_{\text {rel }}=-1 / 4$. This proves part (1) of the theorem.

(a ii) $\lambda_{r e l} \in\left(-\frac{1}{4}, 0\right)$ and $\lambda_{\text {rel }} \neq-\frac{1}{2 l(l+1)}$

In this case, the following holds:

$$
\text { for } \begin{aligned}
\lambda_{r e l} & \in\left(-\frac{1}{4}, 0\right), \lambda_{\text {rel }} \neq-\frac{1}{2 l(l+1)}, \\
w^{0} & =-\frac{\Omega C}{2\left(\frac{1}{2}+2 \lambda_{r e l}\right)} \psi_{10} .
\end{aligned}
$$

(a iii) $\lambda_{\text {rel }} \geq 0$

Since all eigenvalues of $G$ are negative, the kernel of $\left[G-2 \lambda_{r e l}\right]$ is empty for $\lambda_{\text {rel }}>0$. Thus, the solution of (22) is easily found by setting $w^{0}=k \psi_{10}$ :

$$
k=-\frac{\Omega C}{\left(1+4 \lambda_{\text {rel }}\right)} .
$$

This implies the result:

$$
\begin{aligned}
& w^{0}=k \psi_{10} \text { with } k \rightarrow 0^{-} \text {as } \lambda_{r} \rightarrow \infty ; \\
& w^{0} \rightarrow-\Omega C \psi_{10} \text { as } \lambda_{\text {rel }} \rightarrow 0^{+} .
\end{aligned}
$$

The special solution $w^{0}=-\Omega C \psi_{10}$ holds when the constraint $V_{\text {rel }}$ is inoperative, and the variational problem is the unconstrained optimization of the energy $H$. Part (2) of the theorem is proved in a(ii) and a(iii).

(b) $\lambda_{\text {rel }} \in\left[-\frac{1}{4}, 0\right)$ and $\lambda_{r e l}=-\frac{1}{2 l(l+1)}$

At the bifurcation values of $\lambda_{r e l}$ in this regime, the following result holds, as is easily ascertained:

$$
\text { for } \begin{aligned}
\lambda_{r e l} & =-\frac{1}{2 l(l+1)} \in\left[-\frac{1}{4}, 0\right), l=2,3, \ldots, \\
w^{0} & =-\frac{\Omega C}{2\left(\frac{1}{2}-\frac{1}{l(l+1)}\right)} \psi_{10}+\sum_{m=-l}^{l} \alpha_{l m} \psi_{l m} .
\end{aligned}
$$

This proves part (3) of the theorem. QED. 


\section{$5 \quad$ Existence and properties of extremals}

The final steps of the Euler-Lagrange Multiplier Method compute the value of the multiplier $\lambda_{r e l}$ by applying the fixed value of the enstrophy $Q_{r e l}>0$. A consequence of the completion of this circle of calculations is the determination of the physical properties of the extremal relative vorticity $w^{0}$ on the basis of the relative enstrophy $Q_{r e l}$ and the spin rate $\Omega$. Figure 3 shows a plot of (26) and (27).

Lemma 6: The Lagrange Multipliers $\lambda_{\text {rel }}$ of the extremals of the variational problem (11), (12) are given in terms of the spin rate $\Omega \geq 0$ and the relative enstrophy $Q_{\text {rel }}>0$ by

$$
\begin{aligned}
& \lambda_{r e l}^{+}=-\frac{1}{4}\left[1+\frac{\Omega C}{\sqrt{Q_{r e l}}}\right] \\
& \lambda_{r e l}^{-}=-\frac{1}{4}\left[1-\frac{\Omega C}{\sqrt{Q_{r e l}}}\right] .
\end{aligned}
$$

Proof: Substituting the solution (23) of the Euler-Lagrange equation into the constraint in $V_{r}$,

$$
\left\|w^{0}\right\|^{2}=\frac{\Omega^{2} C^{2}}{4\left(\frac{1}{2}+2 \lambda_{r e l}\right)^{2}}=Q_{r e l}>0,
$$

gives the results for $\lambda_{r e l}^{ \pm}$. The remaining special solutions (25) correspond to a countable set of bifurcation values. QED.

The proof of the following statement is left to the reader.

Lemma 7: (1) The first branch of solutions in Lemma 6,

$$
w_{\text {Max }}^{0}=\sqrt{Q_{\text {rel }}} \psi_{10}
$$

corresponding to

$$
\lambda_{r e l}^{+}<-\frac{1}{4}
$$

are associated with solid-body rotation in the direction of spin $\Omega$. In terms of the original kinetic energy

$$
\begin{aligned}
H_{\text {Max }}\left[\alpha_{10} \psi_{10}\right] & =-\Omega^{2} C^{2} \frac{\left(1+8 \lambda_{\text {rel }}^{+}\right)}{16\left(\frac{1}{2}+2 \lambda_{\text {rel }}^{+}\right)^{2}} \\
& =\frac{1}{4} Q_{\text {rel }}+\frac{1}{2} \Omega C \sqrt{Q_{\text {rel }}} .
\end{aligned}
$$


(2) The second branch of solutions

$$
w_{\min }^{0}=-\sqrt{Q_{r e l}} \psi_{10}
$$

associated with

$$
\begin{aligned}
& \lambda_{\text {rel }}^{-} \in\left(-\frac{1}{4}, \infty\right), \\
& \lambda_{\text {rel }}^{-} \neq-\frac{1}{2 l(l+1)}, l=2,3, \ldots,
\end{aligned}
$$

correspond to solid-body rotation opposite the direction of spin. In this case, the original kinetic energy

$$
\begin{aligned}
H_{\min }\left[\alpha_{10} \psi_{10}\right] & =-\Omega^{2} C^{2} \frac{\left(1+8 \lambda_{\text {rel }}^{-}\right)}{16\left(\frac{1}{2}+2 \lambda_{\text {rel }}^{-}\right)^{2}} \\
& =\frac{1}{4} Q_{r e l}-\frac{1}{2} \Omega C \sqrt{Q_{\text {rel }}}
\end{aligned}
$$

for given relative enstrophy $Q_{\text {rel }}$ and spin $\Omega$.

Because the Euler-Lagrange method gives only necessary conditions, to get sufficient conditions for the existence of constrained extremals, it is traditional to use the so-called Direct Method of The Calculus of Variations [21, [20, [23], where it is shown in two parts, that (i) the unconstrained extremals of an augmented objective functional exist, and (ii) these unconstrained extremals are the constrained extremals of the original objective functional. This classical approach is presented in a sequel [24. The approach in this paper of using the infinite dimensional geometry of the energy and relative enstrophy manifolds, can be viewed as an alternative rigorous method for proving the existence of constrained extremals in the coupled fluid-sphere model.

The Euler-Lagrange method gives necessary conditions for extremals of a constrained problem. Sufficient conditions for the existence of extremals can nonetheless be found from the geometrical basis of the method itself. An extremal must be contained in the set of points $p$ with common tangent spaces of the level surfaces of $H$ and $\Gamma$, but only those points $p$ where one level surface remains on the same side of the other level surface in a full neighborhood of $p$ correspond to constrained energy maximizers or minimizers. Of those points, if moreover, the level surface of $H$ is on the outside 
of the surface of $\Gamma$ in a full neighborhood of $p_{M a x}$, with respect to the point $p_{o}=(-\Omega C, 0, \ldots, 0)$ in the subspace of $L_{2}\left(S^{2}\right)$ defined by (14), then $p_{\text {Max }}$ is a constrained energy maximum. If on the other hand, the level surface of $H$ is on the inside of the surface of $\Gamma$ in a full neighborhood of $p_{\min }$, with respect to the point $p_{o}=(-\Omega C, 0, \ldots, 0)$, then $p_{\min }$ is a constrained energy minimum.

All of the above Lemmas and Theorems form that part of variational analysis known as Necessary Conditions. We will use these facts and lemma 3 in the proof of the following existence result which gives Sufficient Conditions for constrained extremals in terms of the geometry of the objective and constraint functionals. Figure 5 depicts case (1) in Theorem 8; figure 6 depicts case $(2 \mathrm{C})$ and figures $7 \mathrm{a}$ and $7 \mathrm{~b}$ show the retrograde solid-body state as constrained local minimum and maximum respectively in both case $(2 \mathrm{~A})$ and $(2 \mathrm{~B})$.

Theorem 8: (1) The first branch of solutions $w_{\text {Max }}^{0}=\sqrt{Q_{\text {rel }}} \psi_{10}$ in Lemmas 6 and 7 are global energy maximizers for any relative enstrophy $Q_{\text {rel }}$ and spin $\Omega$.

(2) For the second branch of solutions $w_{\min }^{0}=-\sqrt{Q_{\text {rel }}} \psi_{10}$ in Lemmas 6 and 7 , the following statements hold:

(A) If relative enstrophy is large compared to spin, i.e.,

$$
Q_{r e l}>4 \Omega^{2} C^{2}
$$

then $w_{\min }^{0}$ is a special saddle point, in the sense that, it is a local energy maxima in all eigen-directions except for $\operatorname{span}\left\{\psi_{1, \pm 1}\right\}$, where it is a local minima.

(B) If relative enstrophy satisfies

$$
\Omega^{2} C^{2}<Q_{r e l}<4 \Omega^{2} C^{2}
$$

then $w_{\min }^{0}$ is a constrained energy saddle point.

(C) If relative enstrophy is small compared to spin, i.e.,

$$
Q_{\text {rel }}<\Omega^{2} C^{2}
$$

then $w_{\min }^{0}$ is a constrained energy minima.

Proof: (1) From the eigenvalues $\lambda_{l m}=-l(l+1)$ and the fact that the spherical harmonics $\psi_{l m}$ diagonalizes the energy $H$, and Lemma 3 (cf. eqn. 
(17)), we deduce that $H$ is positive-definite in $L_{2}\left(S^{2}\right)$, and its level surfaces are infinite dimensional unbounded ellipsoids centered at $p_{o}=(-\Omega C, 0, \ldots 0)$,

with the shortest semi-major axes (of equal lengths)

in $\operatorname{span}\left\{\psi_{10}, \psi_{11}, \psi_{1,-1}\right\}$;

and

all remaining semi-major axes (associated with

azimuthal wavenumber $l$ greater than 1)

have lengths $L(l)$ that are quadratic in $l$

and independent of the wavenumber $m$.

The level surfaces of relative enstrophy are non-compact concentric spheres centered at 0 in $L^{2}\left(S^{2}\right)$,

$$
\|w\|_{2}^{2}=\sum_{l m} \alpha_{l m}^{2}=Q_{r e l}>0
$$

Together this implies that the level surface of $H$ lies on the outside (wrt $p_{o}$ ) of the relative enstrophy level surface for fixed $Q_{\text {rel }}$ in a neighborhood of the point $w_{M a x}^{0}\left(Q_{r e l)}=+\sqrt{Q_{r e l}} \psi_{10}\right.$. This proves that $w_{\text {Max }}^{0}\left(Q_{r e l}\right)$ is a global constrained energy maximizer.

(2) Using the same nomenclature in (D), we determine that when $Q_{r e l}<$ $\Omega^{2} C^{2}$, at the common point $w_{\text {min }}^{0}=-\sqrt{Q_{\text {rel }}} \psi_{10}$, the relative enstrophy hypersphere lies on the outside of the energy ellipsoid wrt $p_{o}$; indeed both surfaces are locally convex with respect to their respective centers. This implies that $w_{\text {min }}^{0}$ is a constrained energy minimum in the case (34).

When $Q_{r e l}>4 \Omega^{2} C^{2}$, it is natural to separate the common tangent space of the ellipsoid and the sphere at $w_{\min }^{0}$ into two orthogonal parts, namely,

$$
\text { (a) } \operatorname{span}\left\{\psi_{1, \pm 1}\right\},(b) \operatorname{span}\left\{\psi_{l m}, l>1\right\} \text {. }
$$

¿From the property (35) and (32), we deduce that the semi-major axes in $\operatorname{span}\left\{\psi_{10}, \psi_{11}, \psi_{1,-1}\right\}$ of the energy ellipsoid at $w_{\text {min }}^{0}$, have equal lengths

$$
L=\left|-\sqrt{Q_{r e l}}+\Omega C\right|>\Omega C .
$$

The enstrophy sphere at $w_{\text {min }}^{0}$ has radii of equal lengths

$$
L_{r e l}=\left|-\sqrt{Q_{r e l}}\right|>2 \Omega C \text {. }
$$


From the fact that the center of this ellipsoid is at $p_{o}=-\Omega C \psi_{10}$ while the sphere is at the origin, it follows that

$$
L<L_{r e l}
$$

This means that in (a) $\operatorname{span}\left\{\psi_{1, \pm 1}\right\}$ of the common tangent space (37) at $w_{\text {min }}^{0}$, this ellipsoid is inside the sphere wrt $p_{o}$, which implies that $w_{\min }^{0}$ is a local energy minimum there. And using (36), we deduce that, in the orthogonal complement, part (b) of this common tangent space (37) at $w_{\text {min }}^{0}$, the ellipsoid is outside the sphere wrt $p_{o}$, which implies that $w_{\min }^{0}$ is a local energy maximum there. Thus, $w_{\min }^{0}$ is a special saddle point in the case (32), in the sense that, apart from $\operatorname{span}\left\{\psi_{1, \pm 1}\right\}$, it is a local maximum.

When $\Omega^{2} C^{2}<Q_{\text {rel }}<4 \Omega^{2} C^{2}$, it follows from property (35) and the unboundedness property (36) of the energy ellipsoid, that there is a critical value of the azimuthal wavenumber $l_{\text {crit }}$, such that in part

$$
\text { (a) } \operatorname{span}\left\{\psi_{l m}, l \leq l_{\text {crit }}\right\}
$$

of the common tangent space at $w_{\min }^{0}$, the ellipsoid is inside the sphere wrt $p_{o}$; and in the orthogonal complement

$$
\text { (b) } \operatorname{span}\left\{\psi_{l m}, l>l_{\text {crit }}\right\} \text {, }
$$

the ellipsoid is outside the sphere wrt $p_{o}$. Thus, $w_{\text {min }}^{0}$ is a constrained energy saddle point in the case (33). QED

\section{Nonlinear Stability Analysis}

The linear stability of the steady-states $w_{\text {Max }}$ and $w_{\min }$ can be read off the second variation of the augmented energy functional $E_{r e l}$. We are interested in proving a stronger result, namely the nonlinear or Lyapunov stability of the energy extremals. In principle this could be done using the geometric (convexity) properties of the relative enstrophy and energy manifolds, recalling that the former is a sphere in $L^{2}\left(S^{2}\right)$ and the latter is an "ellipsoid" in the same Hilbert space. Due to the infinite-dimensionality of $L^{2}\left(S^{2}\right)$, and the consequent non-compactness of the enstrophy manifold, this is a delicate undertaking. 
Instead, we propose to follow the method discussed in detail in [17, [11, [12]. The first step consists of the identification of two quadratic functionals $Q_{1}$ and $Q_{2}$ in the perturbation $\Delta w$ such that

$$
\begin{aligned}
& Q_{1}(\Delta w) \leq H\left[w_{M a x}+\Delta w\right]-H\left[w_{M a x}\right]-D H_{M a x} \cdot \Delta w \\
& Q_{2}(\Delta w) \leq \Gamma\left[w_{M a x}+\Delta w\right]-\Gamma\left[w_{M a x}\right]-D \Gamma_{M a x} \cdot \Delta w
\end{aligned}
$$

Let $\left\{\bar{\alpha}_{l m}\right\}$ denote the perturbation Fourier coefficient based on the steadystate $w_{\text {Max }}=\sqrt{Q_{\text {rel }}} \psi_{10}$, that is, the steady state is given by

$$
\begin{aligned}
\alpha_{10}\left(w_{\text {Max }}\right) & =\sqrt{Q_{\text {rel }}} ; \\
\alpha_{\text {lm }}\left(w_{\text {Max }}\right) & =0 \text { for all other modes. }
\end{aligned}
$$

It is natural and convenient to choose the positive definite quadratic forms

$$
\begin{aligned}
Q_{1}\left(\left\{\bar{\alpha}_{l m}\right\}\right) & \equiv H\left[w_{\text {Max }}+\Delta w\right]-H\left[w_{\text {Max }}\right]-D H_{\text {Max }} \cdot \Delta w \\
& =\frac{\bar{\alpha}_{10}^{2}}{4}-\frac{1}{2} \sum_{l m \neq 10} \frac{\bar{\alpha}_{l m}^{2}}{\lambda_{l m}} \geq 0 ; \\
Q_{2}\left(\left\{\bar{\alpha}_{l m}\right\}\right) & \equiv \Gamma\left[w_{\text {Max }}+\Delta w\right]-\Gamma\left[w_{\text {Max }}\right]-D \Gamma_{\text {Max }} \cdot \Delta w \\
& =\sum \bar{\alpha}_{l m}^{2} \geq 0 .
\end{aligned}
$$

Then, $Q_{1}+Q_{2}$ is positive definite, and is clearly a norm for the perturbation $\Delta w$ since $Q_{2}(\Delta w)=\|\Delta w\|_{2}^{2}$. Indeed, it is the energy-enstrophy norm of Arnold.

From the fact that at steady-state $w_{\text {Max }}, D(H+\Gamma)\left(w_{\text {Max }}\right) \cdot \Delta w=0$, it follows that

$$
\begin{aligned}
\left(Q_{1}+Q_{2}\right)\left(\left\{\bar{\alpha}_{l m}(t)\right\}\right) & \equiv(H+\Gamma)\left[w_{\text {Max }}+\Delta w(t)\right]-(H+\Gamma)\left[w_{\text {Max }}\right] \\
& =(H+\Gamma)\left[w_{\text {Max }}+\Delta w(0)\right]-(H+\Gamma)\left[w_{\text {Max }}\right] \\
& \leq \Delta E(0)
\end{aligned}
$$

that is, the sum $Q_{1}+Q_{2}$ is conserved along trajectories of the BVE. Thus, we conclude that trajectories that start near $w_{\text {Max }}$ will remain nearby for all time, that is, $w_{\text {Max }}$ has been shown to be Lyapunov stable.

The same arguments go through for the energy minimizer $w_{\min }$ when the relative enstrophy $Q_{r e l}<\Omega^{2} C^{2}$. And for the saddle point $w_{\min }$ when $Q_{\text {rel }}>4 \Omega^{2} C^{2}$, a modified argument where perturbations are restricted to 
be in the orthogonal complement of $\operatorname{span}\left\{\psi_{10,}, \psi_{1, \pm 1}\right\}$ in $L^{2}\left(S^{2}\right)$, proves that $w_{\text {min }}$ has some degree of nonlinear stability. This completes the proof of

Theorem 9: The global energy maximizer $w_{\text {Max }}$ is Lyapunov stable under all conditions of spin, energy and relative enstrophy. The constrained energy minimizer $w_{\min }$ is Lyapunov stable in the low energy, low enstrophy regime, that is, when $Q_{r e l}<\Omega^{2} C^{2}$. If $Q_{\text {rel }}>4 \Omega^{2} C^{2}$ (high enstrophy, high energy regime), then the saddle point $w_{\min }$ is nonlinearly stable to all perturbations with azimuthal wavenumber $l \geq 2$. In the intermediate regime, $\Omega^{2} C^{2}<Q_{r e l}<4 \Omega^{2} C^{2}, w_{\min }$ is unstable.

\section{$7 \quad$ Discussion and conclusions}

From the point of view of applications to planetary atmospheres, the physical consequences of theorems 5, 8 and 9 is embodied in the following over-arching statement:

There is a relative enstrophy threshold value $\Omega^{2} C^{2}$ such that below it, the constrained energy extremals consist of the counter-rotating local energy minimizer state $w_{\min }^{0}=-\sqrt{Q_{\text {rel }}} \psi_{10}$ and the pro-rotating global energy maximizer state $w_{\text {Max }}^{0}=+\sqrt{Q_{\text {rel }}} \psi_{10}$. When relative enstrophy exceeds this value, the pro-rotating global energy maximizer state $w_{\text {Max }}^{0}=+\sqrt{Q_{\text {rel }}} \psi_{10}$ persists but the counter-rotating state $w_{\mathrm{min}}^{0}$ becomes a constrained saddle point.

In other words, the counter-rotating state is a nonlinearly stable energy extremal only for low relative enstrophy and low kinetic energy. The nonlinearly stable counter-rotating steady state $w_{\min }^{0}=-\sqrt{Q_{\text {rel }}} \psi_{10}$ changes stability to a constrained saddle point via a saddle-node bifurcation when the rate of spin $\Omega$ decreases past the threshold value

$$
\Omega_{o}=\frac{\sqrt{Q_{r e l}}}{C}
$$

with relative enstrophy fixed at $Q_{\text {rel }}$. When the relative enstropy is large enough compared to the spin rate $\Omega>0$, that is, $Q_{\text {rel }}>\Omega^{2} C^{2}$, the only nonlinearly stable steady state in the model is the global energy maximizer $w_{\text {Max }}^{0}=\sqrt{Q_{\text {rel }}} \psi_{10}$, with the caveat that for $Q_{\text {rel }}>4 \Omega^{2} C^{2}$, the counterrotating state $w_{\min }^{0}$ is nonlinearly stable to zonally-symmetric perturbations.

Thus, for any relative enstrophy and any given spin rate $\Omega>0$, a superrotational Lyapunov stable steady state exists in the coupled model. When 
$Q_{\text {rel }}<\Omega^{2} C^{2}$, both the global energy maximizer and global energy minimizer are Lyapunov stable solid-body rotational states.

The range of phenomena associated with the study of atmospheric superrotation such as pertaining to the Venusian atmosphere (cf. [13] and references therein) is very complex. While we do not claim that our theory and results explain the whole complex range of phenomena associated with superrotation, by going to a model of a barotropic fluid coupled to an infinitely massive rotating sphere - the fluid component exchanges angular momentum as well as kinetic energy with the sphere - we have obtained rigorous results that are directly related to atmospheric super-rotation in both the qualitative and quantitative sense.

We will compare our results here with those from simulations of the General Circulation Model (GCM) as well as those obtained from intermediate level models. The starting point of the body of work based on the GCM is a multi-layer stratified rotating flow model. Furthermore, the system modelled is usually a damped driven one with differential solar radiation and Ekman damping. The best phenomenological results to date - using the GCM - are therefore necessarily based on intensive numerical simulations.

For the purpose of this comparison, numerical simulations of the terrestrial GCM show that super (sub)-rotational flows behave like saturated asymptotic steady states of a complex damped driven system (cf. [13] and ref. therein). The super-rotating state appears to be associated only with sufficiently energetic flows in these simulations. Moreover, the basin of attraction of these flows can be reasonably characterized by only a few initial quantities such as energy and angular momentum, and the super (sub) rotational end states appear to be quite robust in a numerical sense. The super-rotational state is taken to be the one where the fluid component has positive net angular momentum - in the direction of the sphere's spin - relative to the frame in which the sphere is fixed. The vertically averaged barotropic component of the flow in a damped driven multi-layered atmospheric system therefore have asymptotic quasi-steady states which are super and sub-rotational states near to the basic zonal states $\pm \psi_{10}$ [13. Recent numerics (cf. [13] and references therein) confirm the super-rotational state is more relevant to the atmosphere of Venus and is associated only with sufficiently high kinetic energy; the sub-rotational state arises only for lower energies in for instance, the atmosphere of Titan [13].

A more direct comparison can be made between our detailed results and those reported by Yoden and Yamada [5]. In particular, they found robust 
relaxation to a prograde solid-body state for small to intermediate values of the planetary spin, and to a retrograde solid-body state when the planetary spin is large relative to the kinetic energy in the barotropic flow. The first is consistent with our first prediction that the prograde solid-body state arises only when the rest frame kinetic energy of the flow is high enough relative to the planetary spin, or equivalently, for a given range of kinetic energy, it is allowed only for relatively small planetary spins. The second is partially consistent with our second prediction that the retrograde solid-body state is nonlinearly stable only for planetary spins that are large in comparison to the relative enstrophy, if one allows for the fact that the large anticyclonic polar vortex state is a superposition of mainly the retrograde solid-body state and some zonally symmetric spherical harmonics $\psi_{l 0}$ with wavenumber $l<5$.

A related point vortex formulation for the inviscid dynamics of a rotating barotropic vorticity field has been recently reported by Newton et al in [18]. 
Figure 1: Graph of energy $H$ vs. coordinates $k=\alpha_{10}$ of extremals as in (18).
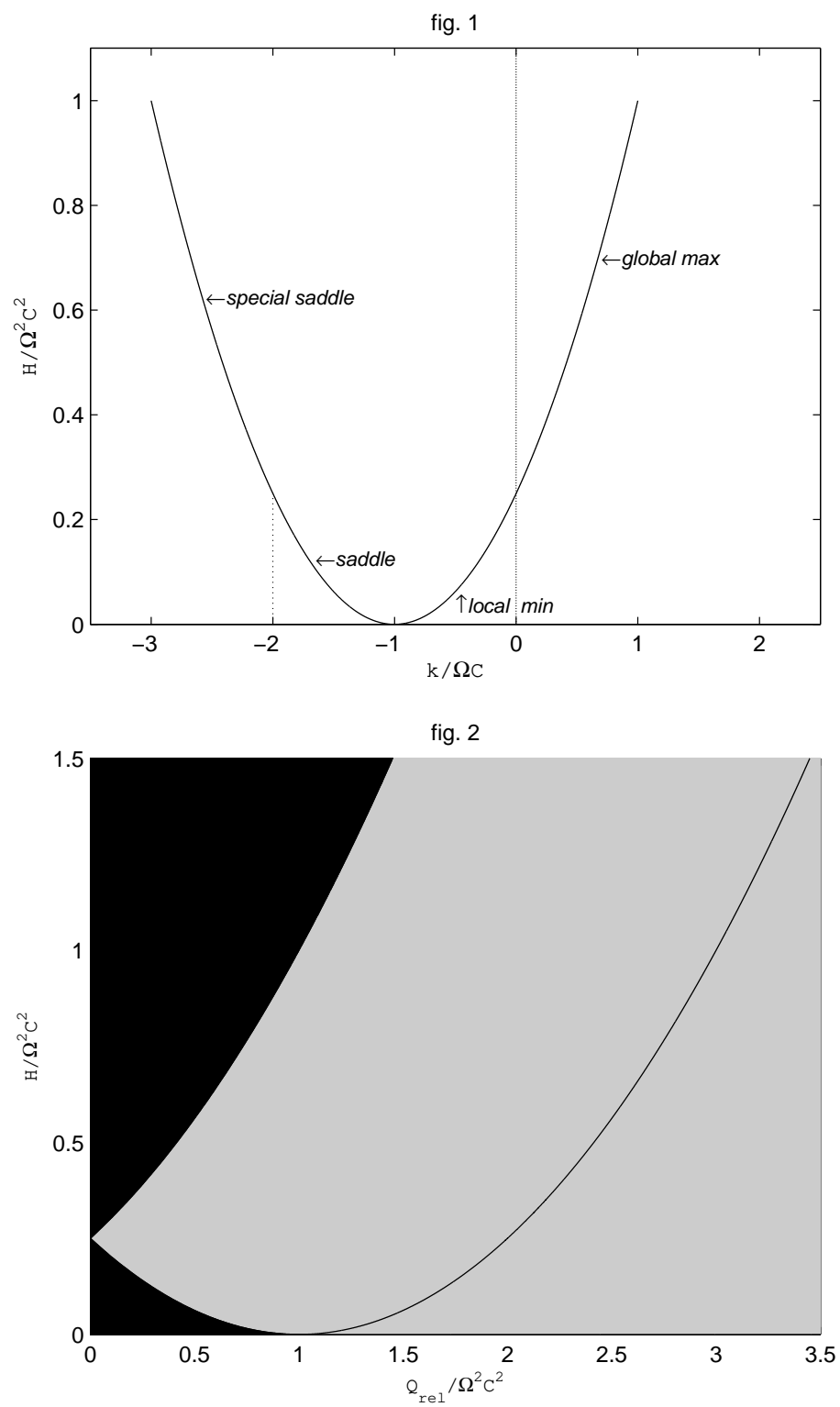

Figure 2: Energy $H$ - relative enstrophy $Q_{\text {rel }}$ space; black region denotes unpermitted values; gray region denotes non-extremal permitted values; curves denote values at extremals as in (19). 
Figure 3: Graph of Lagrange Multipliers $\lambda_{\text {rel }}^{ \pm}$vs. square-root of relative enstrophy, $\sqrt{Q_{\text {rel }}}$ for fixed spin $\Omega>0$ as in (26) and (27).
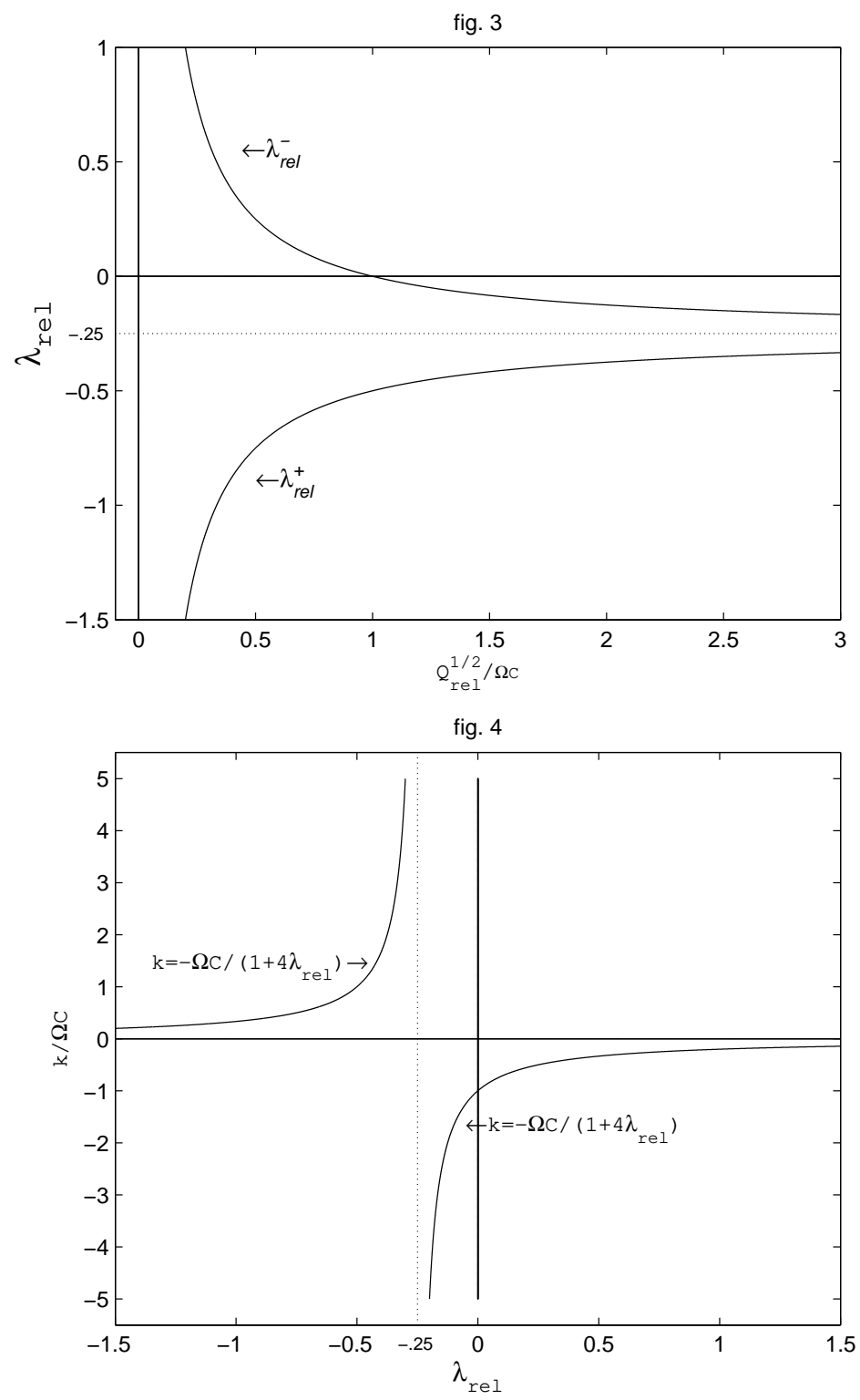

Figure 4: Graph of extremal coordinates $k$ vs. Lagrange Multipliers $\lambda_{\text {rel }}^{ \pm}$for fixed spin $\Omega>0$ as in (23) and (24). 
Figure 5: Projections of energy ellipsoid and enstrophy sphere showing the common tangent at global maximizer $w_{\text {Max }}^{0}$ when energy exceeds $H_{c}$.
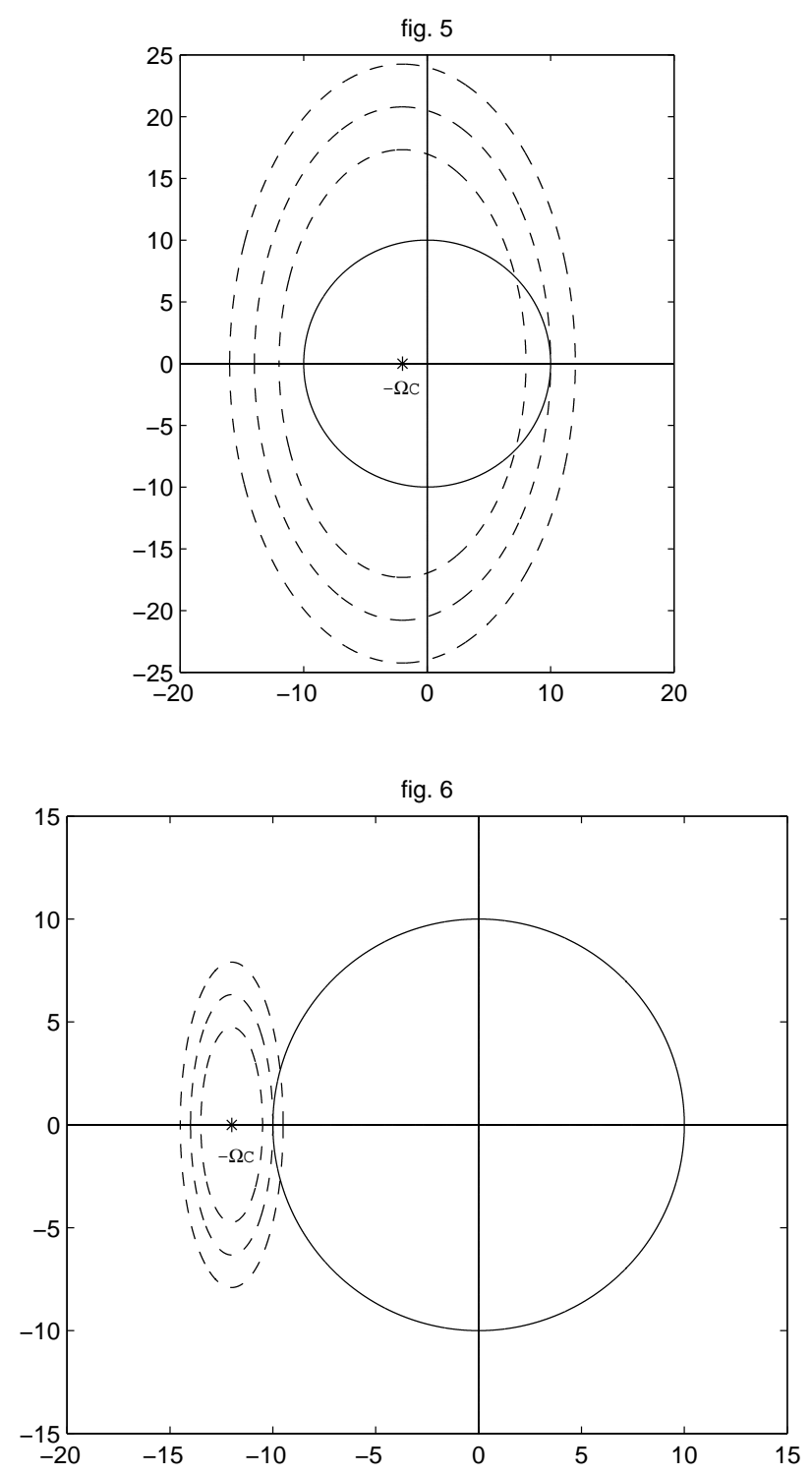

Figure 6: Projections of energy ellipsoid and enstrophy sphere showing the common tangent at local minimizer $w_{\text {min }}^{0}$ when (34) holds. 
Figure 7: (a) Projections of energy ellipsoid and enstrophy sphere showing the saddle point $w_{\text {min }}^{0}$ as local minimum when (133) or (32) holds; (b) Projections of energy ellipsoid and enstrophy sphere showing the saddle point $w_{\text {min }}^{0}$ as local maximum
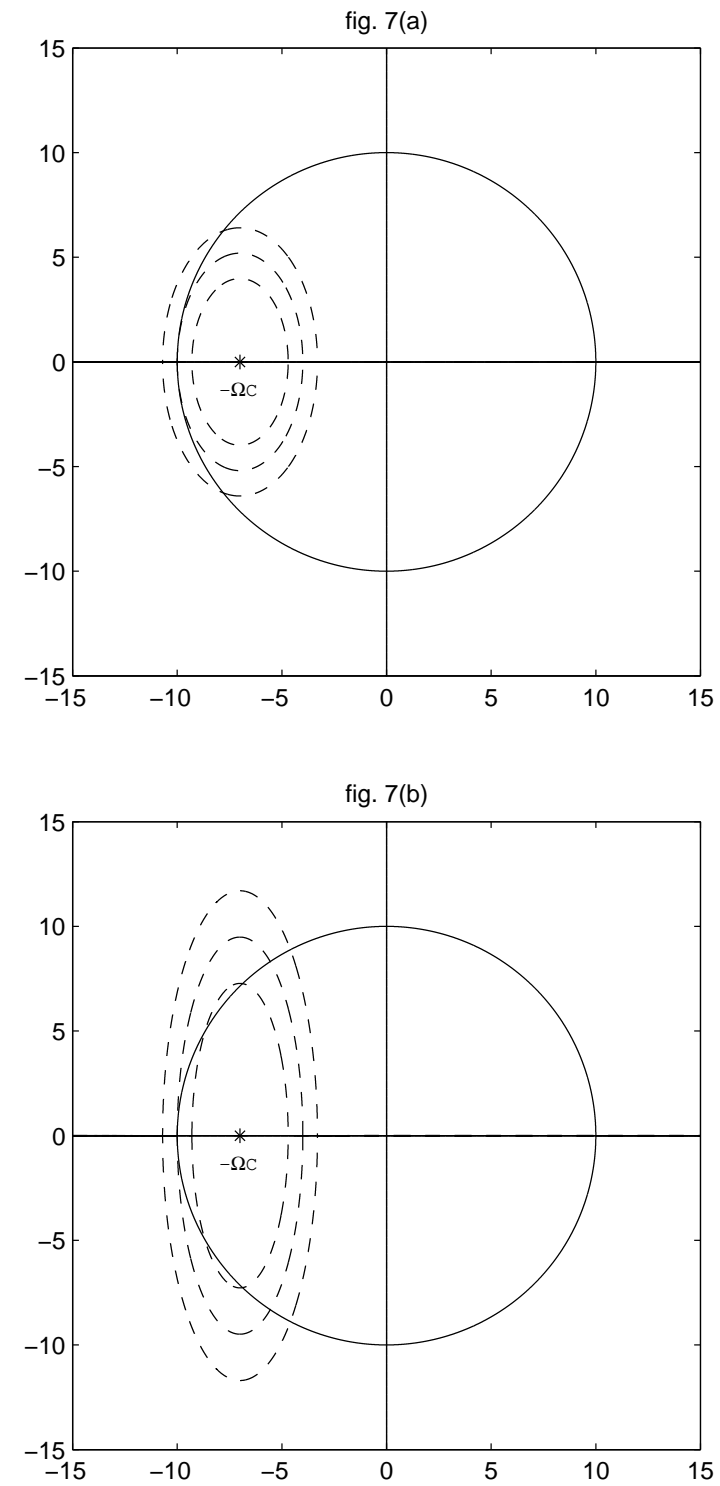


\section{References}

[1] T. G. Shepherd, Non-ergodicity of inviscid two-dimensional flow on a beta-plane and on the surface of a rotating sphere, J. Fluid Mech., 184, 289-302, 1987.

[2] C.C. Lim, Extremal free energy in a simple mean field theory for a Coupled Barotropic Fluid - rotating solid sphere system, to appear DCDS - A 2007.

[3] $\mathrm{Mu} \mathrm{Mu}$ and T.G. Shepherd, On Arnol'd's second nonlinear stability theorem for two-dimensional quasi-geostrophic flow, Geophys.Astrophys.Fluid Dyn. 75 21-37.

[4] J. Cho and L. Polvani, The emergence of jets and vortices in freely evolving, shallow-water turbulence on a sphere, Phys Fluids 8(6), 1531 - 1552, 1995.

[5] S. Yoden and M. Yamada, A numerical experiment on $2 D$ decaying turbulence on a rotating sphere, J. Atmos. Sci., 50, 631, 1993

[6] C. Foias and R. Saut, Indiana U. Math Journ 33, 457 - , 1984.

[7] R. Fjortoft, Application of integral theorems in deriving criteria of stability for laminar flows and for the baroclinic circular vortex, Geofys. Publ. 17(6), 1, 1950.

[8] R. Fjortoft, On the changes in the spectral distribution of kinetic energy for 2D non-divergent flows, Tellus, 5, 225 - 230, 1953.

[9] C. Leith, Minimum enstrophy vortices, Phys. Fluids, 27, 1388 - 1395, 1984.

[10] D. Wirosoetisno and T.G. Shepherd, On the existence of 2D Euler flows satisfying energy-Casimir criteria, Phys.Fluids 12, 727, 2000.

[11] V.I. Arnold, Conditions for nonlinear stability of stationary plane curvilinear flows of an ideal fluid, Sov. Math Dokl., 6, 773-777, 1965.

[12] V.I. Arnold, On an a priori estimate in the theory of hydrodynamical stability, AMS Translations, Ser. 2, 79, 267-269, 1969. 
[13] A. Del Genio, W. Zhuo and T. Eichler, Equatorial Superrotation in a slowly rotating GCM: implications for Titan and Venus, Icarus 101, 117, 1993.

[14] S.J.Chern, Math Theory of the Barotropic Model in GFD, PhD thesis, Cornell University, 1991.

[15] R.H. Kraichnan, Statistical dynamics of two-dimensional flows, J. Fluid Mech. 67, 155-175, 1975.

[16] C. C. Lim, Energy maximizers and robust symmetry breaking in vortex dynamics on a nn-rotating sphere, SIAM J. Appl Math, 65(6), 20932106, 2005.

[17] D. Holm, J. Marsden, T. Ratiu and A. Weinstein, Nonlinear stability of fluid and plasma equilibria, Physics Report 123, 1-116, 1985.

[18] P. Newton and H. Shokraneh, The N-Vortex Prob on a Rotating Sphere, Proc. R. Soc. London Series A, 462, 149 - 169, 2006

[19] P.B. Rhines, Waves and turbulence on a beta plane, J. Fluid Mech., 69(3), 417-443, 1975.

[20] I. Ekeland and R. Temam, Convex Analysis and Variatonal Problems, North-Holland, 1976.

[21] D. Smith, Variational Methods in Optimization, Dover

[22] K.K. Tung, Barotropic instability of zonal flows, J. Atmos. sc., 38, 308 - 321, 1981.

[23] P. D. Lax, Functional Analysis, Wiley-Interscience, 2002.

[24] C.C. Lim and Junping Shi, The role of higher vorticity moments in a variational formulation of a Barotropic Vorticity Model on the rotating sphere, submitted 2005.

\section{List of Figure Captions:}

Figure 1. Graph of energy $H$ vs. coordinates $k=\alpha_{10}$ of extremals as in (18). 
Figure 2. Energy $H$ - relative enstrophy $Q_{r e l}$ space; black region denotes unpermitted values; gray region denotes non-extremal permitted values; curves denote values at extremals as in (19).

Figure 3. Graph of Lagrange Multipliers $\lambda_{\text {rel }}^{ \pm}$vs. square-root of relative enstrophy, $Q_{\text {rel }}$ for fixed spin $\Omega>0$ as in (26) and (27).

Figure 4. Graph of extremal coordinates $k$ vs. Lagrange Multipliers $\lambda_{\text {rel }}^{ \pm}$ for fixed spin $\Omega>0$ as in (23) and (24).

Figure 5. Projections of energy ellipsoid and enstrophy sphere showing the common tangent at global maximizer $w_{\text {Max }}^{0}$ when energy exceeds $H_{c}$.

Figure 6. Projections of energy ellipsoid and enstrophy sphere showing the common tangent at local minimizer $w_{\min }^{0}$ when (34) holds.

Figure 7a. Projections of energy ellipsoid and enstrophy sphere showing the saddle point $w_{\min }^{0}$ as local minimum when (33) or (32) holds.

Figure 7b. Projections of energy ellipsoid and enstrophy sphere showing the saddle point $w_{\min }^{0}$ as local maximum. 